\title{
Effect of Processing Parameters on Performance of Spray-Deposited Organic Thin-Film Transistors
}

\author{
Jack W. Owen, ${ }^{1}$ Natalia A. Azarova, ${ }^{1}$ Marsha A. Loth, ${ }^{2}$ Markos Paradinas, ${ }^{3}$ Mariona Coll, ${ }^{3}$ \\ Carmen Ocal, ${ }^{3}$ John E. Anthony, ${ }^{2}$ and Oana D. Jurchescu ${ }^{1}$
}

${ }^{1}$ Department of Physics, Wake Forest University, Winston-Salem, NC 27109, USA

${ }^{2}$ Department of Chemistry, University of Kentucky, Lexington, KY 40506, USA

${ }^{3}$ Institut de Ciència de Materials de Barcelona, ICMAB-CSIC, Campus UAB, 08193-Barcelona, Spain

Correspondence should be addressed to Oana D. Jurchescu, jurcheod@wfu.edu

Received 1 February 2011; Accepted 14 March 2011

Academic Editor: Marisol Reyes-Reyes

Copyright (C) 2011 Jack W. Owen et al. This is an open access article distributed under the Creative Commons Attribution License, which permits unrestricted use, distribution, and reproduction in any medium, provided the original work is properly cited.

\begin{abstract}
The performance of organic thin-film transistors (OTFTs) is often strongly dependent on the fabrication procedure. In this study, we fabricate OTFTs of soluble small-molecule organic semiconductors by spray-deposition and explore the effect of processing parameters on film morphology and device mobility. In particular, we report on the effect of the nature of solvent, the pressure of the carrier gas used in deposition, and the spraying distance. We investigate the surface morphology using scanning force microscopy and show that the molecules pack along the $\pi$-stacking direction, which is the preferred charge transport direction. Our results demonstrate that we can tune the field-effect mobility of spray-deposited devices two orders of magnitude, from $10^{-3} \mathrm{~cm}^{2} / \mathrm{Vs}$ to $10^{-1} \mathrm{~cm}^{2} / \mathrm{Vs}$, by controlling fabrication parameters.
\end{abstract}

Organic semiconductors have emerged as novel materials for electronic devices which combine the advantages of low cost with high diversity and compatibility with a broad range of substrates $[1,2]$. A wide variety of polymers and smallmolecule organic semiconductors have been developed and investigated in devices such as organic thin-film transistors (OTFTs), organic photovoltaic cells (OPVs), and organic light-emitting diodes (OLEDs) $[3,4]$. Single crystals of small-molecule organic semiconductors have demonstrated impressive properties, such as high mobility [5-8], metalliclike behavior [9], and long-range exciton diffusion [10]. Remarkable transistor properties were reported in solutiondeposited soluble small molecules having strong $\pi$-stacking interactions [11-15]. Solution-processable small-molecule organic semiconductors offer attractive properties which are of interest across the spectrum of organic electronic devices from OPV, to OTFTs and OLEDs. These attributes include low-complexity synthesis and purification procedures, superior charge carrier mobilities, and better chemical stability compared to polymers. Moreover, they are monodisperse, and parameters such as polydispersity, regioregularity, and batch-to-batch variations are not an issue, which greatly improves reproducibility. Fabrication methods based on solution deposition, such as spin-coating, drop-casting, or ink-jet printing are of particular interest as they meet the manufacturing requirements for low-cost electronics. Unfortunately, these methods are not necessarily compatible with large-area electronics, and given the strong dependence of film electrical properties on microstructure, which in turn is severely driven by processing details, it is not clear that the impressive performance derived from these materials can be reproduced over larger areas. We have recently demonstrated high-performance small-molecule organic semiconductor TFTs fabricated by spray-coating [16]. This method offers fast deposition over large areas at low cost and thus meets manufacturing requirements for large-area electronics. Our results on spray-deposited transistors have demonstrated transistor performance similar to spin-coated devices, while utilizing 20 times smaller amount of organic semiconductor than the wasteful spin-coating procedure.

In this paper, we will explore the effect of processing parameters on the performance of spray-deposited OTFTs 


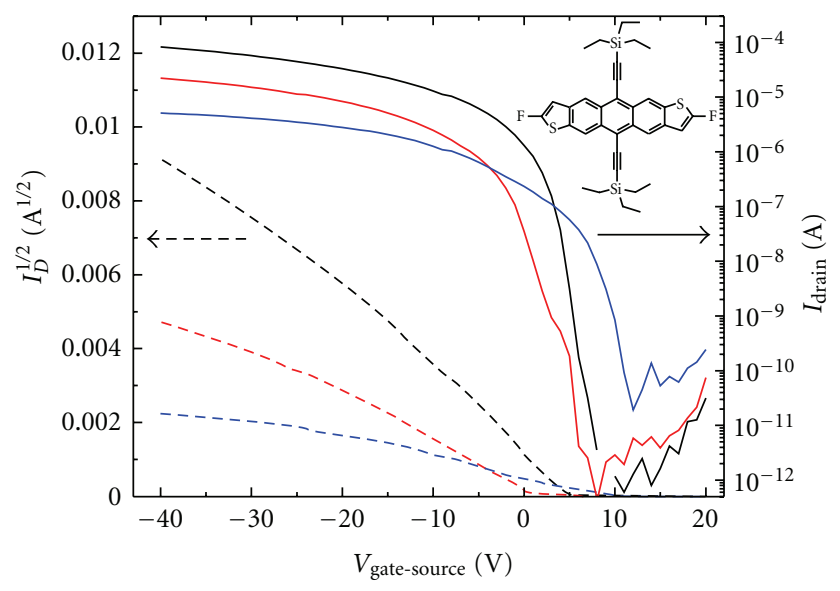

FIGURE 1: Current-voltage characteristics (drain current $I_{\mathrm{D}}$ versus gate-to- source voltage $V_{\mathrm{GS}}$ in saturation regime, $V_{\mathrm{DS}}=-40 \mathrm{~V}$ ) for diF TES ADT devices sprayed from chlorobenzene (black), toluene (red) and dichlorobenzene (blue) solutions. The right axis corresponds to the solid lines and represents $\log \left(I_{\mathrm{D}}\right)$, and the left axis corresponds to the dotted lines and shows the $\sqrt{ } I_{\mathrm{D}}$.

and demonstrate that the field-effect mobility can span several orders of magnitude in thin films of nominally the same material upon choice of solvent used for fabrication or variations in pressure of the carrier gas, spraying distance, and solution concentration. The dependence of device parameters on processing conditions may represent a serious issue for the reproducibility and reliability of OTFTs, and for this reason a good insight into the effect of these parameters should offer better control over device performance. By using local probe techniques, we demonstrate that the morphology of the spray-deposited devices showing the best performance is in agreement with that of spin-coated devices, and for this reason comparable performance is achieved.

In order to investigate the effect of processing parameters on device structure, we have fabricated OTFTs by spray coating a solution of 2,8-difluoro-5,11-bis(triethylsilylethynyl) anthradithiophene(diF-TESADT, chemical structure shown in the inset of Figure 1) over prefabricated device structures. These test beds consist of heavily doped Si gate electrode, $200 \mathrm{~nm}$ thermally grown $\mathrm{SiO}_{2}$, and e-beam evaporated $\mathrm{Ti} / \mathrm{Au}$ $(5 \mathrm{~nm} / 45 \mathrm{~nm})$ source and drain contacts. The substrates were cleaned with hot acetone, isopropyl alcohol, followed by UV ozone cleaning and DI water rinse. The contacts were chemically modified using PFBT(pentafluorobenzene thiol)selfassembled monolayer (SAM), which was demonstrated to improve charge injection and film morphology $[3,11]$. The organic semiconductor was dissolved in a solvent, and the solution was sprayed in atmospheric conditions, by using an Iwata Kustom TH K9200 air brush, under pressurized high purity argon, held at a controlled distance above the device substrate. The air brush was held in a fixed position, aiming perpendicular to the substrate, and the spraying process took about $20 \mathrm{~s}$. More details on fabrication were reported elsewhere [16]. In Figure 1, we present the electrical properties of diF-TES ADT TFTs, with the organic film being spray-coated from three different solvents: chlorobenzene (black), toluene (red), and dichlorobenzene (blue). All electrical measurements were performed in ambient atmosphere. On the right axis, in solid lines, we show the evolution of the drain current $\left(I_{\mathrm{D}}\right)$ with the gate-to-source voltage $\left(V_{\mathrm{GS}}\right)$ in the saturation regime (drain-to-source voltage $V_{\mathrm{DS}}$ $=-40 \mathrm{~V})$. On the left axis, in dotted lines, we present the evolution of the $\sqrt{ } I_{\mathrm{D}}$ versus $V_{\mathrm{GS}}$ for the same devices. The latter plot was used to determine the field-effect mobility in the saturation regime, from the following expression:

$$
I_{\mathrm{D}}=\frac{1}{2} \frac{W}{L} C_{\mathrm{i}} \cdot \mu \cdot\left(V_{\mathrm{GS}}-V_{\mathrm{T}}\right)^{2}
$$

where $W$ and $L$ are the channel width and length, respectively, $C_{\mathrm{i}}$ is the gate oxide capacitance per unit area $\left(C_{\mathrm{i}}=1.7\right.$. $\left.10^{-4} \mathrm{~F} / \mathrm{m}^{2}\right), \mu$ represents the field-effect mobility, and $V_{\mathrm{T}}$ is the threshold voltage. The channel geometries are $L=$ $20 \mu \mathrm{m}$ and $L=1000 \mu \mathrm{m}$ for all three devices presented in Figure 1, and the corresponding mobilities are $\mu=0.3 \mathrm{~cm}^{2} / \mathrm{Vs}$ for the film deposited from a chlorobenzene solution, $\mu=$ $0.1 \mathrm{~cm}^{2} / \mathrm{Vs}$ for the toluene sample, and $\mu=0.01 \mathrm{~cm}^{2} / \mathrm{Vs}$ for the dichlorobenzene sample. These represent typical values obtained in over 10 OTFTs for each solvent. Because the semiconductor does not incorporate solvent into its crystal packing, the differences in the value of mobility come from the fact that the three solvents present different evaporation rates, leading to different crystal formation environment, which is mirrored in the electronic properties. High-vaporpressure solvents, such as toluene, promote faster solvent evaporation, which substantially reduces the ability of the semiconductor molecules to organize in a highly ordered fashion. On the contrary, low-vapor-pressure solvents, such as chlorobenzene, promote slower evaporation rates and thus longer crystal formation time, allowing for molecular arrangement prior to transition to the solid phase. For this reason, a lower mobility is measured in toluene (vapor pressure $\sim 24 \mathrm{mmHg}$ at $22^{\circ} \mathrm{C}$ ) than in chlorobenzene (vapor pressure $\sim 10 \mathrm{mmHg}$ at $22^{\circ} \mathrm{C}$ ). This figure also points out that the vapor pressure of the solvent does not always scale with mobility and is thus not sufficient to describe the differences measured in electrical properties: the film deposited from dichlorobenzene exhibited the lowest mobility $(\mu=$ $\left.0.01 \mathrm{~cm}^{2} / \mathrm{Vs}\right)$, in spite of the fact that this solvent has the lowest vapor pressure $\left(\sim 1.5 \mathrm{mmHg}\right.$ at $\left.22^{\circ} \mathrm{C}\right)$. Optical inspection of the film deposited from dichlorobenzene points out that the film presents poor connectivity between the crystalline domains and the ring-like-shape "coffee stain" features. This result suggests that with spray-coating, film formation is more complex, and it is critically affected by other factors such as the surface energy of the substrate, and the wettability of the solvent. When the solvent evaporates, the solution becomes supersaturated and crystals are formed on the substrate via a nucleation and growth mechanism. This is a very dynamic and complex process, driven by the interplay between solvent/substrate, solvent/vapor, solute/substrate, and solute/solvent interactions. Figure 1 also points out that not only the mobility, but also the threshold voltage of devices is affected by the choice of solvent: OTFTs deposited from dichlorobenzene and chlorobenzene showed positive 


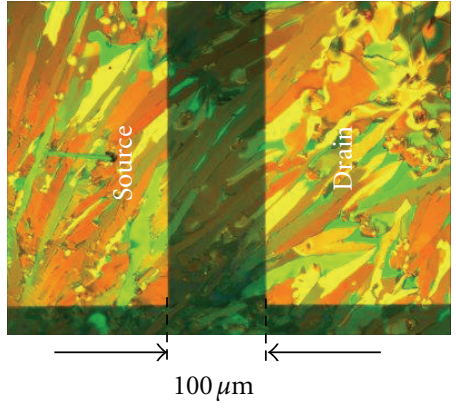

(a)

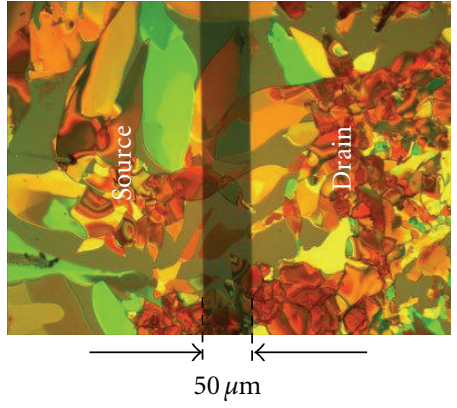

(b)

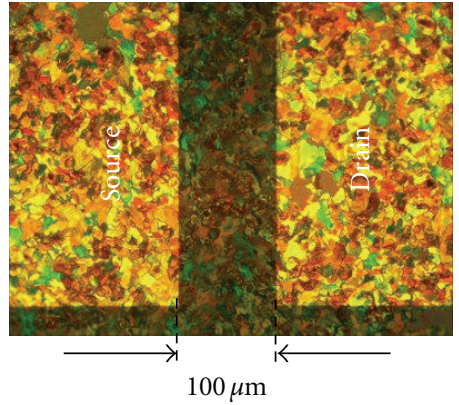

(c)

FIGURE 2: Optical micrograph of TFTs sprayed from a solution in chlorobenzene, under three argon pressures: (a) 5 psi, (b) 10 psi, and (c) 25 psi. The source and drain gold contacts are indicated.

TABle 1: Field-effect mobility for diF TES-ADT devices spraycoated under various carrying gas pressure.

\begin{tabular}{lccccc}
\hline Ar pressure (psi) & 5 & 9 & 10 & 18 & 25 \\
\hline$\mu$ & 0.15 & 0.03 & 0.04 & 0.05 & 0.08 \\
$\left(\mathrm{~cm}^{2} / \mathrm{Vs}\right)$ & $( \pm 0.05)$ & $( \pm 0.007)$ & $( \pm 0.008)$ & $( \pm 0.02)$ & $( \pm 0.02)$ \\
\hline
\end{tabular}

shifts, possibly due to the polarity of the solvent, in agreement with reports on drop-cast 5,11-bis(triethylsilylethynyl) anthradithiophene [17]. This effect is not as pronounced in the value of the on/off ratio, which is remarkably high for samples sprayed from chlorobenzene (values of $10^{8}$, similar to toluene transistors), but it is clearly observed in dichlorobenzene samples, where it drops to $10^{5}$.

Purity of organic semiconductor is known to critically affect the electronic properties. In pentacene, for example, the reduction of pentacenequinone content from $0.68 \%$ to $0.028 \%$ allowed the demonstration of mobilities as high as $35 \mathrm{~cm}^{2} / \mathrm{Vs}$ and band-like behavior $[5,6]$. For this reason, we have performed the spray coating under high-purity argon, to avoid material degradation and/or the introduction of impurities during deposition. We have observed that the pressure of the carrier gas can play a determining role in the electrical properties of the devices, as it can be observed from Table 1. Here, we present the effect of the argon pressure on the device mobility for devices sprayed from a chlorobenzene solution, with the nozzle placed at distance of $13 \mathrm{~cm}$ from the substrate.

The mobility values reported in Table 1 are averaged over 10 devices. At low carrier gas pressures ( $5 \mathrm{psi})$, the TFTs present the best performance, with an average mobility of $0.15 \mathrm{~cm}^{2} / \mathrm{Vs}$. As the gas pressure is increased, the gas "blows away" the solution from the substrate, resulting in lowerquality films, which is in agreement with the lower values of mobility measured in these films. However, a small increase in mobility is observed when the pressure increases from 9 psi to $25 \mathrm{psi}$. This effect can be understood by a careful examination of the optical images presented in Figure 2. Here, devices fabricated at 5 psi (Figure 2(a)), 10 psi (Figure 2(b)), and $25 \mathrm{psi}$ (Figure 2(c)) are presented. The source and drain contacts are indicated. At low pressures (Figure 2(a)), the film covering the transistor channel consists of large crystallites spanning between the source and drain contacts, in agreement with the high mobility measured here. At higher pressures (Figure 2(b)), the continuity of the film becomes problematic: the crystallites are much smaller and they only cover a fraction of the transistor channel, yielding lower currents. When the pressure is increased even further, in spite of the fact that the grains become even smaller (Figure 2(c)), their density increases, resulting in better surface coverage and improved performance. The coverage obtained for $P=25 \mathrm{psi}$ is similar to that resulting from $5 \mathrm{psi}$, but the mobility mitigates by a factor of two. We believe that this is a result of smaller crystalline domains present in this sample and the increased density of grain boundaries connecting them. This is in agreement with earlier reports that demonstrated significant voltage drops at the grain boundaries present in transistor channels [18] and one order of magnitude larger resistance across the grain boundaries than within the grains measured using conducting probe atomic force microscopy [19]. The films obtained by spraycoating consist of high-mobility regions within crystalline grains and low-mobility regions connecting them, and the field-effect mobility of OTFTs is a weighted sum of the two. The measured mobility is thus a function of the intrinsic mobility of the organic semiconductors (intragrain mobility) as well as the concentration of the grain boundaries (intergrain mobility). Larger grain size is usually beneficial for charge transport, as demonstrated by Lee et al. in 5,11bis(triethylsilylethynyl) anthradithiophene TFTs [20], and this phenomenon can also be observed by comparing the devices deposited at 5 psi and 25 psi, for which the coverage is similar but the grain size is different.

To obtain a better picture of the morphology of the sprayed films, we have performed scanning force microscopy (SFM) and explored structural details such as crystalline quality, film lateral continuity, and the presence of point and extended defects. All SFM measurements were carried out under $\mathrm{N}_{2}$ atmosphere $(\mathrm{RH}<2 \%)$ to diminish any possible humidity effects. Figure 3 shows SFM images (dynamic mode) carried out on a device spray coated from a $1.1 \mathrm{mg} / \mathrm{mL}$ solution in chlorobenzene, at 5 psi argon, from $13 \mathrm{~cm}$, in a region within the organic film between metallic electrodes 


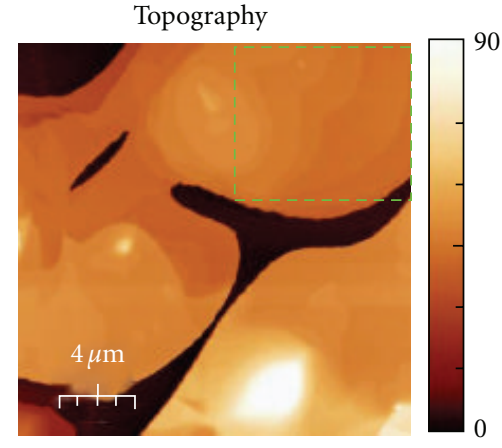

(a)

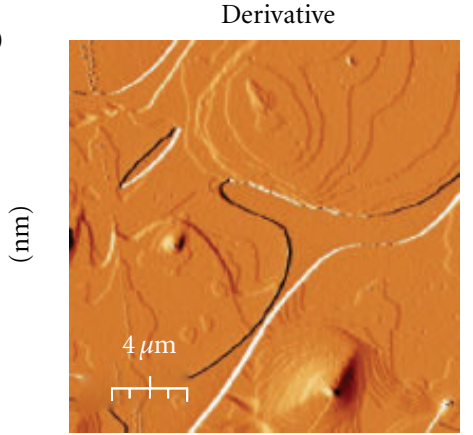

(b)

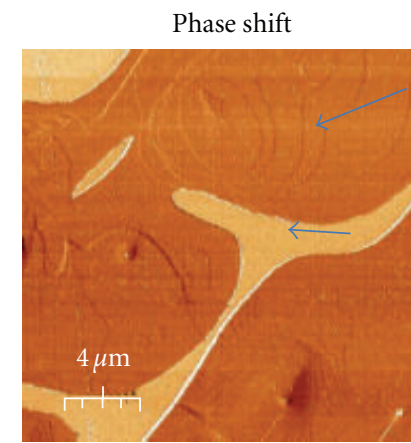

(c)

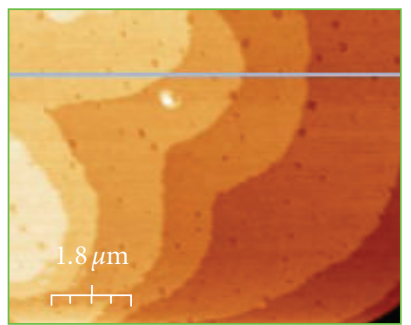

(d)

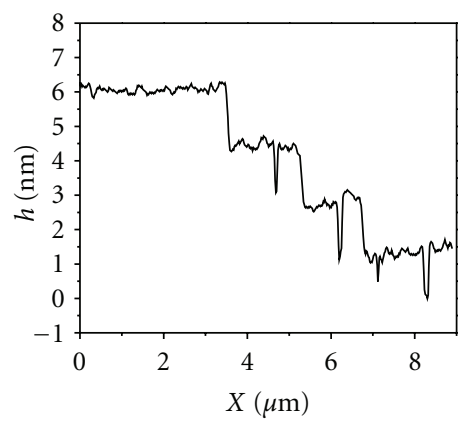

(e)

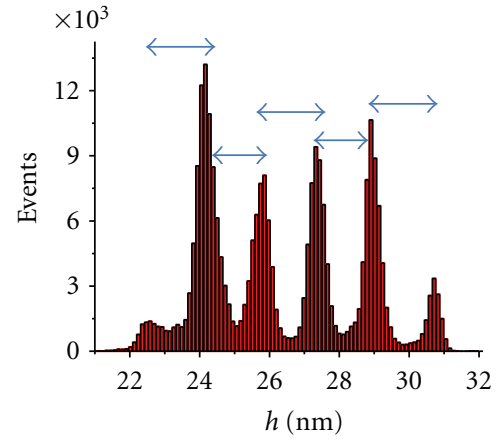

(f)

FIGURE 3: SFM data of a spray-deposited diF-TES ADT, partially covering the interelectrode device region. (a) Topographic image, (b) topography derivative image, (c) phase shift image, (d) magnification topography taken on the dotted area in (a). (e) Line profile taken along the blue segment in (d) crossing three single steps and some one-layer-deep vacancy defects. (f) Height histogram of image (d).

(transistor channel). Regions of well-developed terraces separated by steps $(\mathrm{a}-\mathrm{d})$ indicate the formation of a crystalline organic film. The derivative image in (b) highlights the presence of typical defects (e.g., screw dislocations, vacancy islands) and the pancake morphology of some grains, with up to about 7 different levels in this case. Figure 3(c) shows the phase shift image that will be described in detail later. The image in Figure 3(d) is a zoom into the film region within the dotted green lines in Figure 3(a). The profile (e) taken along the blue segment in the topographic image (d), as well as the height histogram (f) of the whole image, shows a mean step height of $\mathrm{h} \approx 1.65 \mathrm{~nm}$, in agreement with the interlayer separation of single crystals and spincoated diF-TES ADT crystalline films. Thus, we conclude that the growth mode of spray-deposited diF-TES ADT is similar to that of analogous spin-cast films on PFBT-treated substrates, which show a (001) molecular orientation $[3,21]$, with the ADT backbone of the molecules cofacially packed. This molecular arrangement is the most favorable for charge transport, as predicted by the theoretical calculations of Brédas et al. [22], and it is similar to the single-crystal structure, in which mobilities as high as $6 \mathrm{~cm}^{2} / \mathrm{Vs}$ were reported $[23,24]$. This observation is important in its ability to explain the similar mobilities obtained in spraycoated and spin-coated devices, as a result of the same molecular packing. With diF-TES ADT, a mobility in the order of $10^{-3} \mathrm{~cm}^{2} / \mathrm{Vs}$ is measured when the molecules adopt a mixed orientation in the film, and mobilities as high as $0.2-0.5 \mathrm{~cm}^{2} / \mathrm{Vs}$ can be achieved upon surface treatment that induces a (001) molecular orientation [11]. In spite of the fact that the film formation for the sprayed and spincoated samples is very different, with significantly shorter time allowing for solvent evaporation in the spin-coating process, the interactions at the surfaces dominate the filmforming environment, and highly ordered cofacially packed molecules are present in both type of films. The clear two level contrast observed and indicated by blue arrows in the corresponding phase shift image (Figure 3(c)) is directly correlated to the highest topographic difference $(\approx 30 \mathrm{~nm})$ in (a). This excitation frequency contrast is known to come from a difference in total tip-sample interaction at different locations and is a consequence of different local surface properties, for instance due to the presence of different materials, chemical terminations, wetting properties, electrostatic interactions, and so forth. No phase contrast exists between the single-layer terraces of the grains, as otherwise expected from a unique crystallographic termination of the organic film, and the larger phase shift value (i.e., a more dissipative response) at the lower topographic level is most probably due to noncovered substrate regions. In order to elucidate if the differences observed in the phase shift image are due to film discontinuity and verify if these inhomogeneities reach the underlying substrate, the same surface area (containing the two regions of interest) 


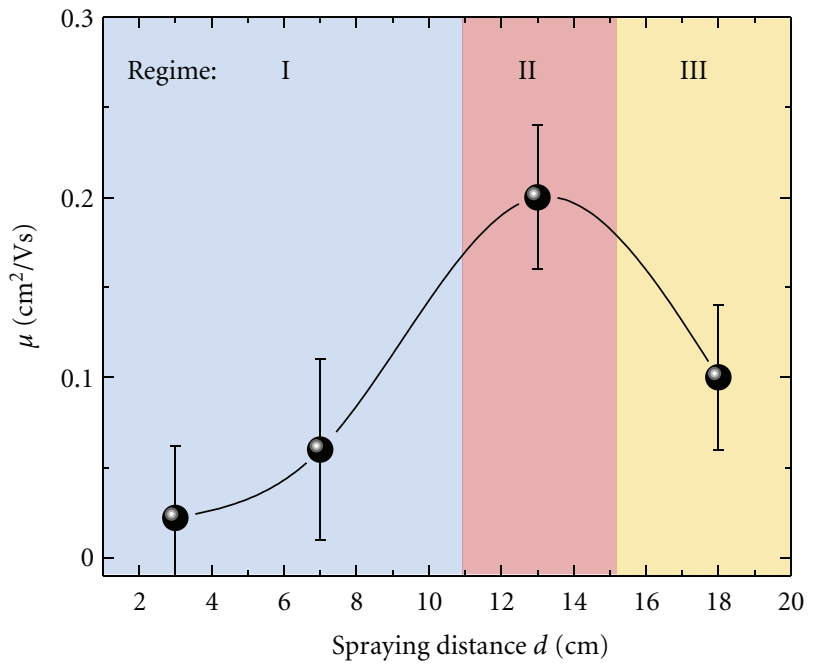

Figure 4: Measured field-effect mobility $(\mu)$ as a function of the spraying distance (d). Three distinct regimes can be identified.

was analyzed using a combination of dynamic operation (phase shift) and contact-mode friction force microscopy, FFM [25] (see Supporting information). A clear contrast between the two regions was observed in the dissipationresponse channels (phase shift and lateral force images). The calculated friction supports the larger dissipation measured on the lower surface level with no differences among the film terrace levels, thus confirming the different tribological behavior of the two regions as corresponding to two different material properties. Moreover, the lower surface level has a root mean squared roughness of about $0.4 \mathrm{~nm}$, as expected from uncovered $\mathrm{SiO}_{2}$ substrate regions.

In this section we were interested in the effect that the spraying distance has on the performance of the devices. Figure 4 presents the evolution of field-effect mobility with the distance between the substrate and the spray nozzle, $d$, when the gas pressure was held constant, $p=5$ psi. This plot indicates three distinct regimes. For short distances (regime I, $0 \mathrm{~cm}<d<11 \mathrm{~cm}$ ), the air brush is too close to the substrate and it has an effect similar to high gas pressure: the solution on the substrate is partially blown away by the incoming flow. This effect becomes less pronounced, and the mobility improves as the distance is increased, reaching the best values for $11 \mathrm{~cm}<d<15 \mathrm{~cm}$ (regime II). With further increase of the spraying distance (regime III, $d>15 \mathrm{~cm}$ ), the mobility drops to lower values, possibly due to lower film crystallinity as a result of the fact that the solvent evaporates before the small aerosolized solution droplets reach the substrate. We expect that the boundaries between the three regimes will shift when a different carrier pressure gas is used, with larger distances being optimal at higher pressures, to minimize the solution removal by the incoming flow.

In summary, we have demonstrated that the nature of solvent, pressure of the carrying gas, and the distance between the substrate and the air brush dramatically affect film forming properties in spray-deposited organic thin-film transistors and that this is reflected in significant variations in device performance, with field-effect mobility varying two orders of magnitude upon using different processing parameters. Our best spray-coated OTFTs have mobility of $0.3 \mathrm{~cm}^{2} / \mathrm{Vs}$ and on/off ratio of $10^{8}$.

\section{Acknowledgment}

The authors thank Dr. David Gundlach from the National Institute of Standards and Technology for supplying the substrates coated with $\mathrm{SiO}_{2}$ and $\mathrm{Au}$.

\section{References}

[1] T. Sekitani, U. Zschieschang, H. Klauk, and T. Someya, "Flexible organic transistors and circuits with extreme bending stability," Nature Materials, vol. 9, no. 12, pp. 1015-1022, 2010.

[2] J. E. Anthony, "The larger acenes: versatile organic semiconductors," Angewandte Chemie - International Edition, vol. 47, no. 3, pp. 452-483, 2008.

[3] D. J. Gundlach, J. E. Royer, S. K. Park et al., "Contactinduced crystallinity for high-performance soluble acenebased transistors and circuits," Nature Materials, vol. 7, no. 3, pp. 216-221, 2008.

[4] G. Dennler, M. C. Scharber, and C. J. Brabec, "Polymerfullerene bulk-heterojunction solar cells," Advanced Materials, vol. 21, no. 13, pp. 1323-1338, 2009.

[5] O. D. Jurchescu, J. Baas, and T. T. M. Palstra, "Effect of impurities on the mobility of single crystal pentacene," Applied Physics Letters, vol. 84, no. 16, pp. 3061-3063, 2004.

[6] O. D. Jurchescu, M. Popinciuc, B. J. Van Wees, and T. T. M. Palstra, "Interface-controlled, high-mobility organic transistors," Advanced Materials, vol. 19, no. 5, pp. 688-692, 2007.

[7] M. E. Gershenson, V. Podzorov, and A. F. Morpurgo, "Colloquium: electronic transport in single-crystal organic transistors," Reviews of Modern Physics, vol. 78, no. 3, pp. 973989, 2006.

[8] J. Takeya, J. Kato, K. Hara et al., "In-crystal and surface charge transport of electric-field-induced carriers in organic singlecrystal semiconductors," Physical Review Letters, vol. 98, no. 19, Article ID 196804, 2007.

[9] I. N. Hulea, S. Fratini, H. Xie et al., "Tunable Fröhlich polarons in organic single-crystal transistors," Nature Materials, vol. 5, no. 12, pp. 982-986, 2006.

[10] H. Najafov, B. Lee, Q. Zhou, L. C. Feldman, and V. Podzorov, "Observation of long-range exciton diffusion in highly ordered organic semiconductors," Nature Materials, vol. 9, no. 11, pp. 938-943, 2010.

[11] O. D. Jurchescu, B. H. Hamadani, H. D. Xiong et al., "Correlation between microstructure, electronic properties and flicker noise in organic thin film transistors," Applied Physics Letters, vol. 92, no. 13, Article ID 132103, 2008.

[12] S. K. Park, J. E. Anthony, and T. N. Jackson, "Solutionprocessed TIPS-pentacene organic thin-film-transistor circuits," IEEE Electron Device Letters, vol. 28, no. 10, pp. 877879, 2007.

[13] S. K. Park, T. N. Jackson, J. E. Anthony, and D. A. Mourey, "High mobility solution processed 6,13-bis(triisopropylsilylethynyl) pentacene organic thin film transistors," Applied Physics Letters, vol. 91, no. 6, Article ID 063514, 2007.

[14] S. K. Park, D. A. Mourey, S. Subramanian, J. E. Anthony, and T. N. Jackson, "High-mobility spin-cast organic thin film transistors," Applied Physics Letters, vol. 93, no. 4, Article ID 043301, 2008. 
[15] T. Uemura, Y. Hirose, M. Uno, K. Takimiya, and J. Takeya, "Very high mobility in solution-processed organic thin-film transistors of highly ordered [1] benzothieno[32b]benzothiophene derivatives," Applied Physics Express, vol. 2, no. 11, Article ID 111501, 2009.

[16] N. A. Azarova, J. W. Owen, C. A. McLellan et al., "Fabrication of organic thin-film transistors by spray-deposition for low-cost, large-area electronics," Organic Electronics: Physics, Materials, Applications, vol. 11, no. 12, pp. 1960-1965, 2010.

[17] C. S. Kim, S. Lee, E. D. Gomez, J. E. Anthony, and Y. L. Loo, "Solvent-dependent electrical characteristics and stability of organic thin-film transistors with drop cast bis(triisopropylsilylethynyl) pentacene," Applied Physics Letters, vol. 93, no. 10, Article ID 103302, 2008.

[18] L. C. Teague, B. H. Hamadani, O. D. Jurchescu et al., "Surface potential imaging of solution processable acene-based thin film transistors," Advanced Materials, vol. 20, no. 23, pp. 45134516, 2008.

[19] T. W. Kelley and C. D. Frisbie, "Gate voltage dependent resistance of a single organic semiconductor grain boundary," Journal of Physical Chemistry B, vol. 105, no. 20, pp. 45384540, 2001.

[20] S. S. Lee, C. S. Kim, E. D. Gomez et al., "Controlling nucleation and crystallization in solution-processed organic semiconductors for thin-film transistors," Advanced Materials, vol. 21, no. 35, pp. 3605-3609, 2009.

[21] R. J. Kline, S. D. Hudson, X. Zhang et al., "Controlling the microstructure of solution-processable small molecules in thin-film transistors through substrate chemistry," Chemistry of Materials, vol. 23, no. 5, pp. 1194-1203, 2011.

[22] J. L. Brédas, D. Beljonne, V. Coropceanu, and J. Cornil, "Charge-transfer and energy-transfer processes in $\pi$ conjugated oligomers and polymers: a molecular picture," Chemical Reviews, vol. 104, no. 11, pp. 4971-5003, 2004.

[23] O. D. Jurchescu, S. Subramanian, R. J. Kline et al., "Organic single-crystal field-effect transistors of a soluble anthradithiophene," Chemistry of Materials, vol. 20, no. 21, pp. 6733-6737, 2008.

[24] O. D. Jurchescu, D. A. Mourey, S. Subramanian et al., "Effects of polymorphism on charge transport in organic semiconductors," Physical Review B - Condensed Matter and Materials Physics, vol. 80, no. 8, Article ID 085201, 2009.

[25] C. Munuera, E. Barrena, and C. Ocal, "Chain-length dependence of metastable striped structures of alkanethiols on Au(111)," Langmuir, vol. 21, no. 18, pp. 8270-8277, 2005. 

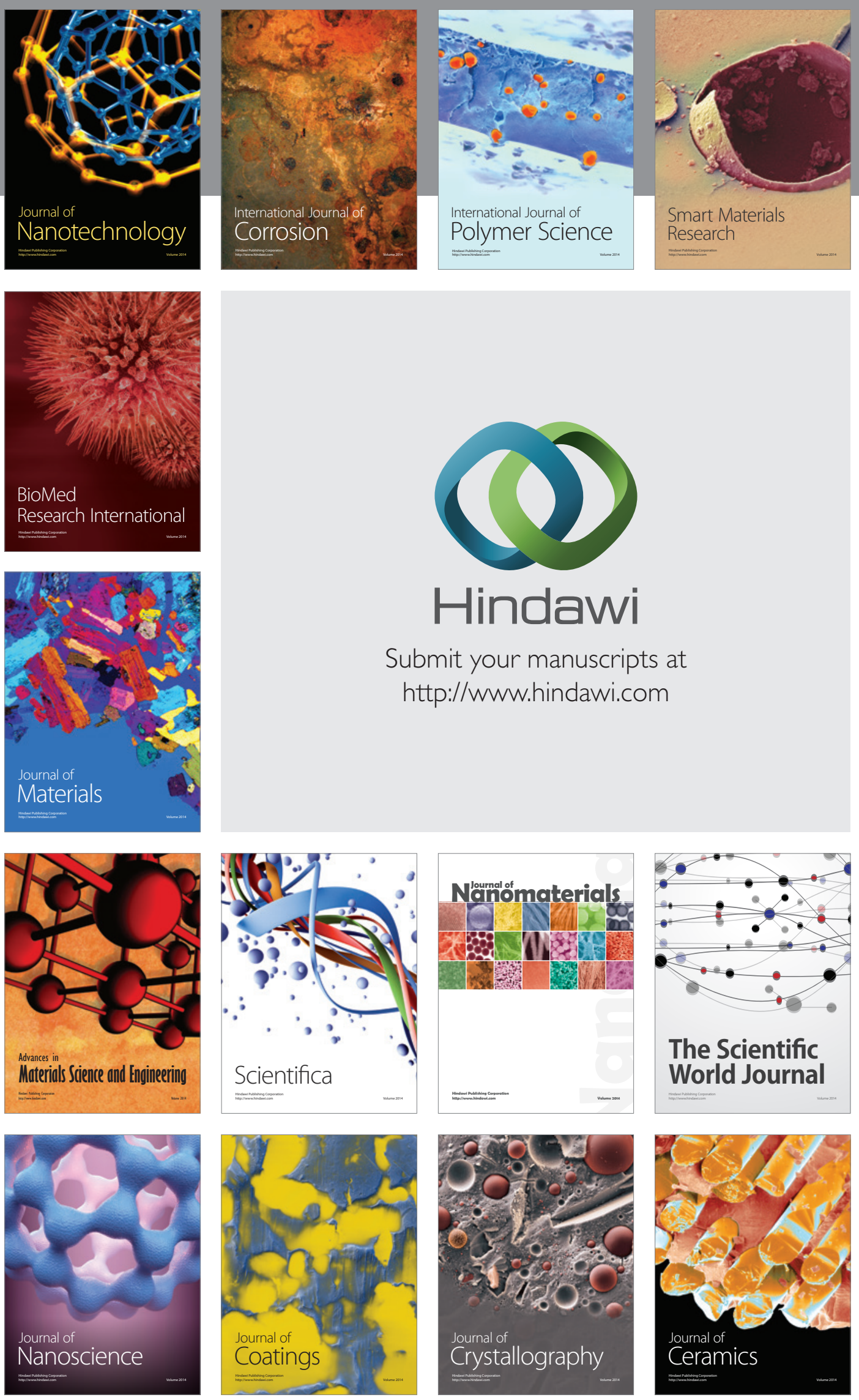

The Scientific World Journal

Submit your manuscripts at

http://www.hindawi.com

\section{World Journal}

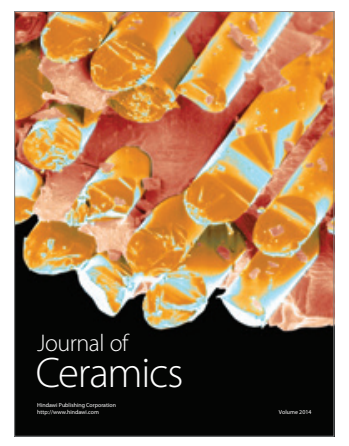

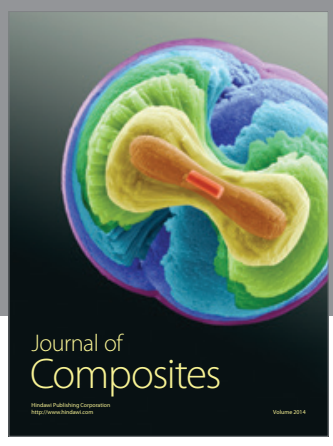
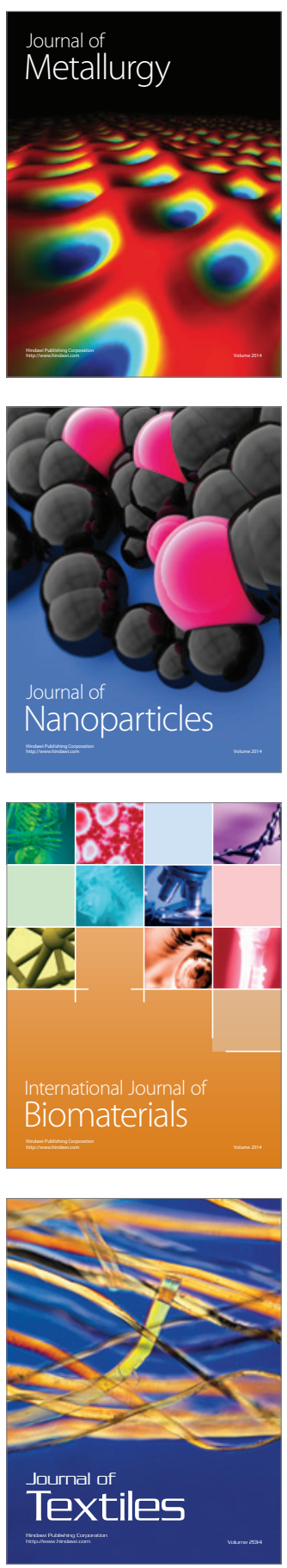